\title{
Electromagnetic Propagation Modeling in Office Environment
}

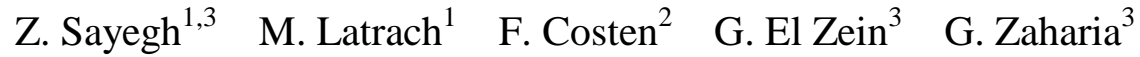

\begin{abstract}
Wireless propagation modeling is more and more needed for optimal indoor coverage in complex environment (such as offices, classrooms, etc.). Thus the need to predict electromagnetic propagation efficiently and accurately in the presence of obstacles, allowing engineers to planning efficiently the communicating devices. This paper will present a rigorous electromagnetic indoor propagation modeling based on the FDTD (3D) method taking into account the presence of various obstacles. A comparison between numerical results obtained by FDTD code and Ray Tracing software will be presented and compared with measurement results. Computational performance efficiency of these methods will also be discussed.
\end{abstract}

\section{INTRODUCTION}

Efficiency and accuracy are increasingly required in wireless propagation modeling in indoor environments for planning better coverage. This coverage is influenced by the geometry and material characteristics of existing obstacles in the environment.

A code based on the FDTD (3D) method has been developed with FORTRAN and adapted to our context of study taking into account the presence of obstacles and their properties[1]-[4].

This code has a capability to produce the electromagnetic fields in time domain at any locations in the environment, field distribution in spatial domain and the relative power distribution.

Many models have been developed and proposed for the prediction of electromagnetic wave propagation (ray tracing, dominant path, COST 231- multi wall ...). However, the calculation using these models for complex environment takes important computing time and requires enormous computing resources. This led us to compare between results obtained by our FDTD code and others obtained by ray tracing software and measurements.

The computational performance efficiency of these methods will also be discussed.

\section{SCENARIO}

A typical office measuring $34 \lambda \times 27 \lambda \times 24 \lambda$ is used for this study [1]-[2], or $\lambda$ is the wavelength, featuring a brick enclosure walls, two metal wardrobes, one metal heater, two metal desks, two computers, two screens, four glass windows and one wooden door (Figure 1). We used monopole omnidirectional antennas with the physical size of $\lambda / 4$ and resonate at $2.4 \mathrm{GHz}$.

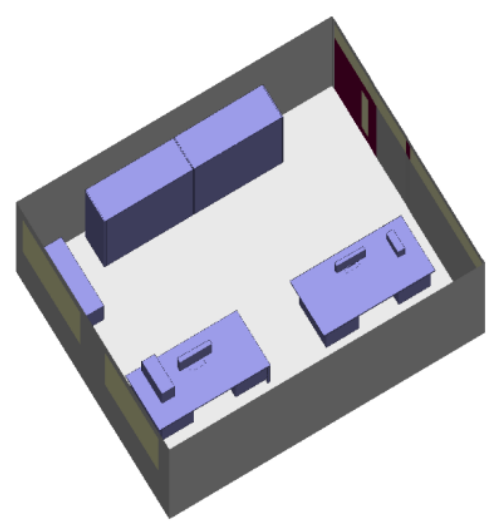

Figure 1: Office environment study.

\section{OFFICE MODELING WITH FDTD CODE}

We start by defining the scenario's geometry with our FDTD code, and then the materials properties (conductivity and permittivity).

We need to define the frequency $(2.4 \mathrm{GHz}$ in our study), the spatial step ( $\lambda / 10$ chosen to be small to get a perfect continuity of space and time and to minimize the errors introduced by the numerical dispersions), the location of transmitting antenna (85 $\mathrm{cm}$ above the floor level), and the output points were we need to get the electromagnetic fields values in time domain (143 points).

Once we run the simulation, the code calculate the electric and magnetic fields in time domain for each spatial step, and produce the field's values for the defined output points in ' $t x t$ ' files.

\footnotetext{
${ }^{1}$ IETR-ESEO, Radio \& Microwave Team, 10 Bd Jeanneteau - CS 90717, 49107 Angers Cedex 2, France, e-mail: zaher.sayegh@eseo.fr.

${ }^{2}$ School of Electric and Electrical Engineering, University of Manchester, U.K.

${ }^{3}$ IETR-INSA de Rennes, Rennes, France.
} 
Figure 2 shows the propagation of electromagnetic waves in the environment for 2000 time steps (or the time step is 24 ps) taking into account the presence of obstacles and the reflections, transmissions and diffractions inside the office.

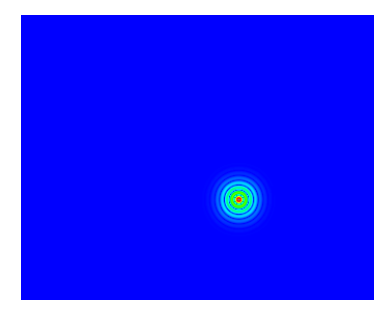

$\mathrm{t}=2.4 \mathrm{~ns}$

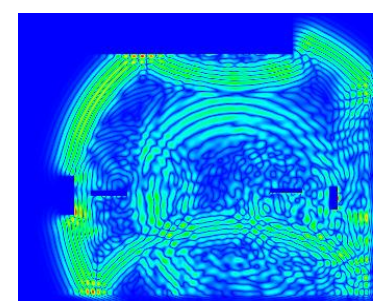

$\mathrm{t}=9.12 \mathrm{~ns}$

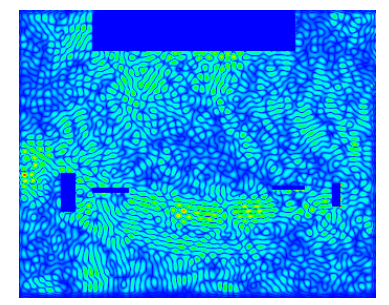

$\mathrm{t}=16.2 \mathrm{~ns}$

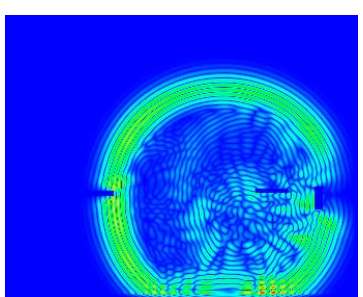

$\mathrm{t}=6.72 \mathrm{~ns}$

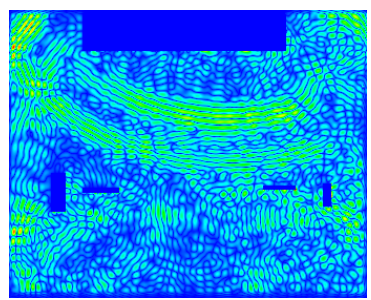

$\mathrm{t}=12.72 \mathrm{~ns}$

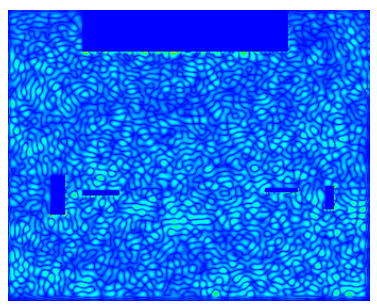

$\mathrm{t}=43.44 \mathrm{~ns}$
Figure 2: Electromagnetic wave propagation in time domain (FDTD code).

In order to compute the radiated power at $2.4 \mathrm{GHz}$, we use the Fourier transform to get the electromagnetic fields in frequency domain, and then we extract $\mathrm{E}_{\mathrm{x}}, \mathrm{E}_{\mathrm{y}}, \mathrm{E}_{\mathrm{z}}, \mathrm{H}_{\mathrm{x}}, \mathrm{H}_{\mathrm{y}}$ and $\mathrm{H}_{\mathrm{z}}$ at $2.4 \mathrm{GHz}$ to calculate the Poynting vector $\mathrm{P}=|\mathrm{E} \times \mathrm{H}|$ [5].

$$
\begin{gathered}
P=\left(\left[\left(E_{y} \cdot H_{z}\right)-\left(E_{z} \cdot H_{y}\right)\right]^{2}+\left[\left(E_{z} \cdot H_{x}\right)-\left(E_{x} \cdot H_{z}\right)\right]^{2}\right. \\
\left.+\left[\left(E_{x} \cdot H_{y}\right)-\left(E_{y} \cdot H_{x}\right)\right]^{2}\right)^{1 / 2}\left(\mathrm{~W} / \mathrm{m}^{2}\right)
\end{gathered}
$$

The repartition of power loss computed by the FDTD code for 143 points is represented in Figure 3. We can see the influence of computers, heater and wardrobes on the radiation of antenna by the dark blue areas.

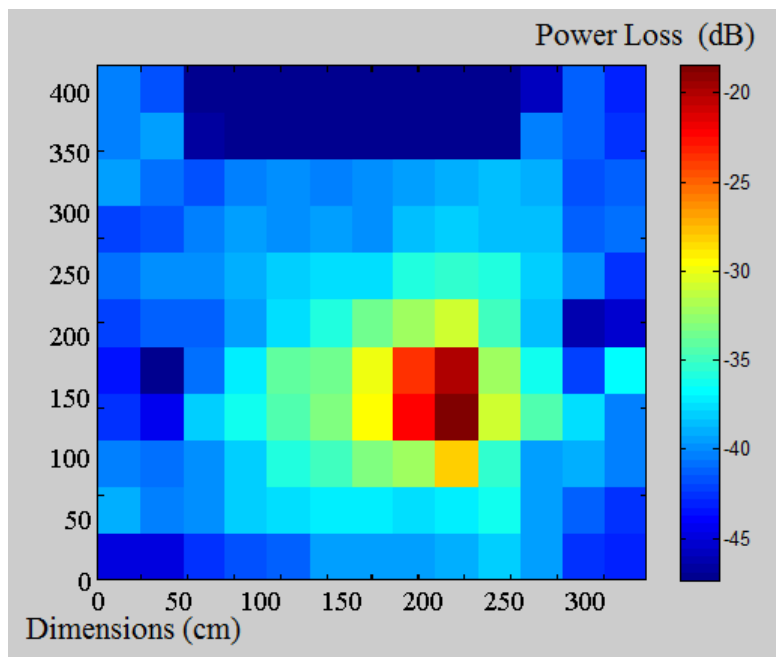

Figure 3: Repartition of Power Loss (dB) obtained by FDTD code.

\section{OFFICE MODELING WITH RAY TRACING}

We define the same scenario with ray tracing; we need to specify the transmitting antenna location and also the receiving antennas positions, and then we choose the number of reflections, transmissions and diffractions, if these number are chosen high we get more accuracy but the computation time increase.

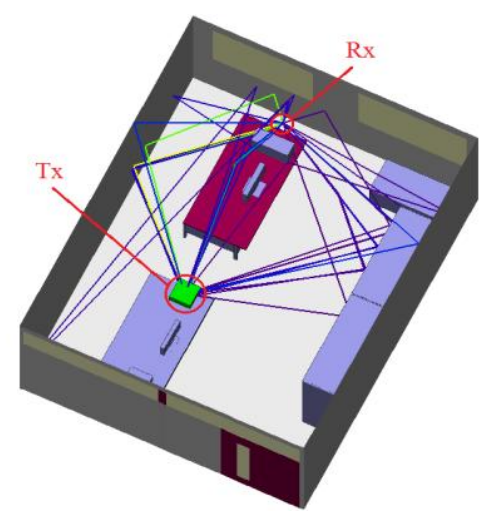

Figure 4: Paths between Tx and Rx obtained by Ray Tracing.

Figure 4 shows the paths between the transmitting antenna and one of the 143 receiving antenna position. We chose two maximum reflections, eight maximum transmissions and one diffraction.

The repartition of power loss computed by the ray tracing method for 143 points is represented in Figure 5. 


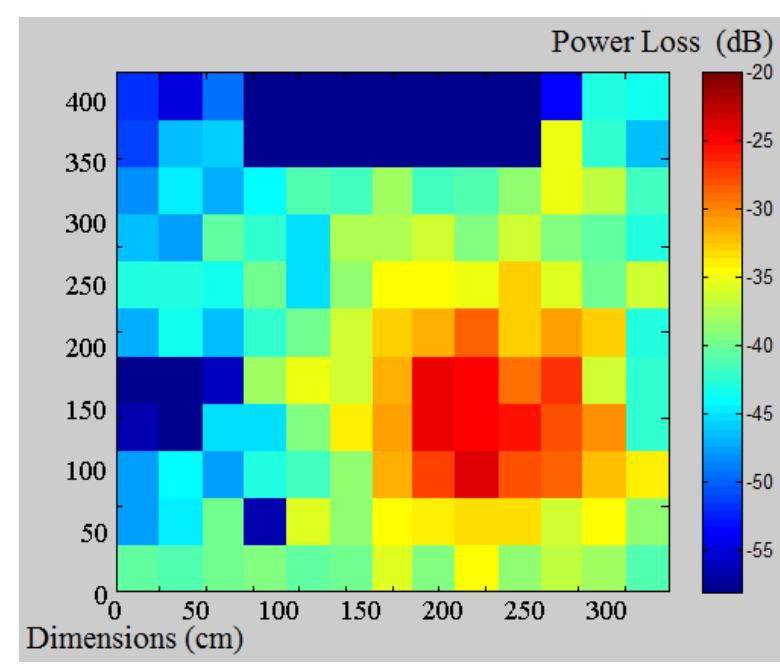

Figure 5: Repartition of Power Loss (dB) obtained by Ray Tracing.

\section{COMPARISON WITH MEASUREMENTS}

In this scenario, we used two monopole omnidirectional antennas $(\lambda / 4)$ resonate at $2.4 \mathrm{GHz}$. The transmitting antenna is placed $10 \mathrm{~cm}$ above the desk as shown in Figure 6, the transmit power of 0 $\mathrm{dBm}$ is emitted by a signal generator which operates at $10 \mathrm{MHz}$ to $20 \mathrm{GHz}$. The received power is measured with a spectrum analyzer $(9 \mathrm{kHz}-3 \mathrm{GHz})$.

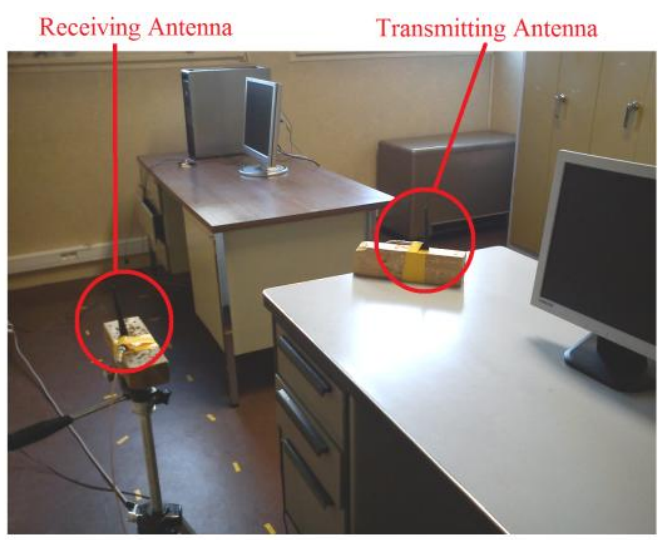

Figure 6: Measurement of antenna radiated power in office environment at $2.4 \mathrm{GHz}$.

The measurements are made in 143 positions separate by $2.5 \lambda$ at the same height of the transmitting antenna (Figure 7).

The repartition of the power loss measured is shown in Figure 8. The power value is the average of 8 calculated values of power for 8 dots placed around each measurement position at a distance of $\lambda / 2$, to reduce the influence of fluctuations due to multipath propagation.

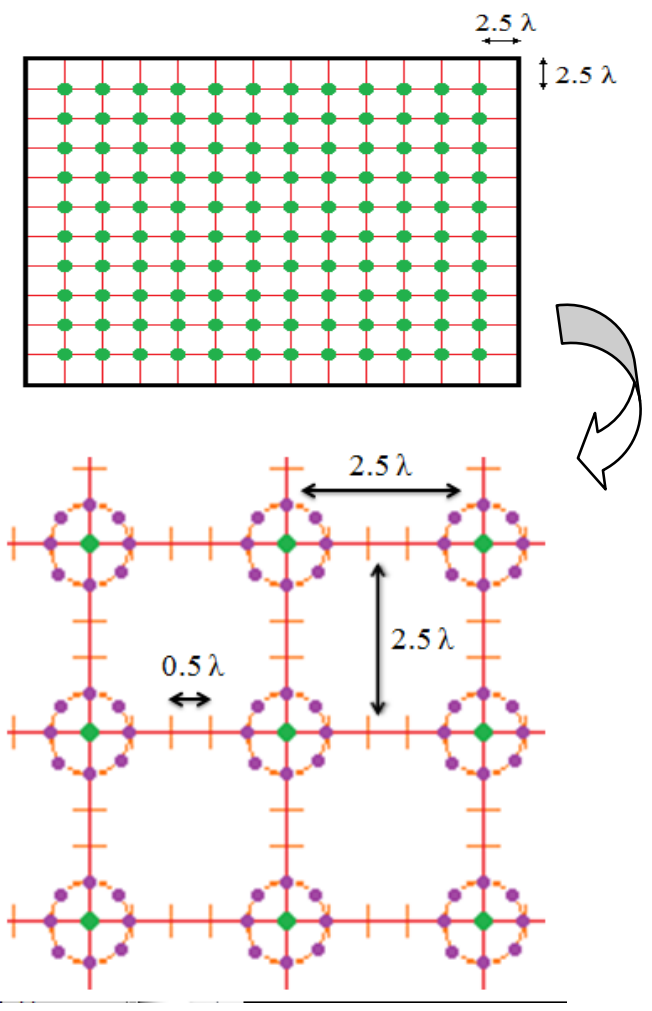

Figure 7: Measurement method used.

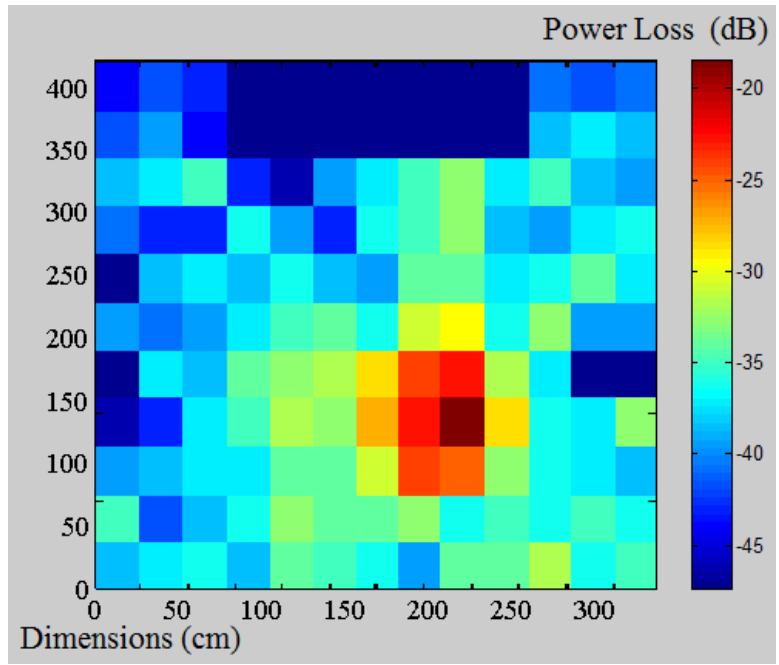

Figure 8: Repartition of Power Loss (dB) obtained by measurements.

\section{COMPARISON BETWEEN RESULTS}

The results obtained by FDTD code and Ray Tracing are compared with measurements results. 
Figure 9 (a) shows the difference between results obtained by ray tracing and measurements and the Figure 9 (b) shows the difference between results obtained by FDTD code and measurements.

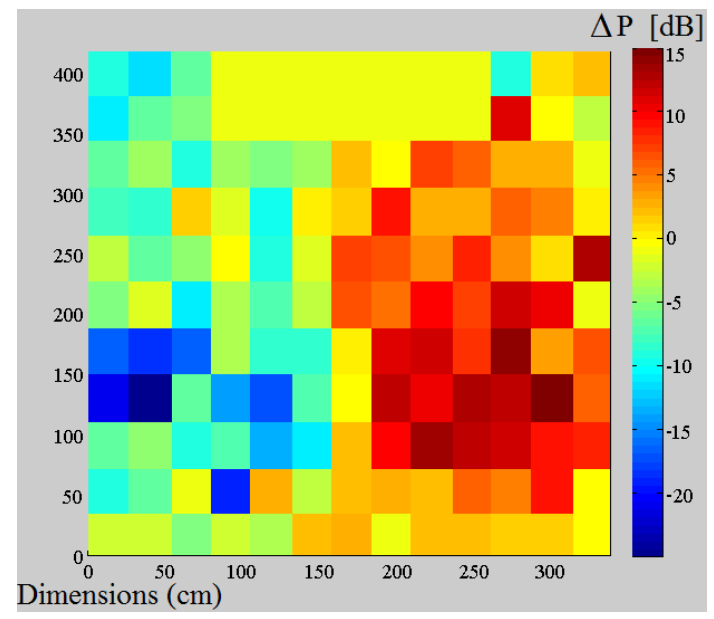

(a)

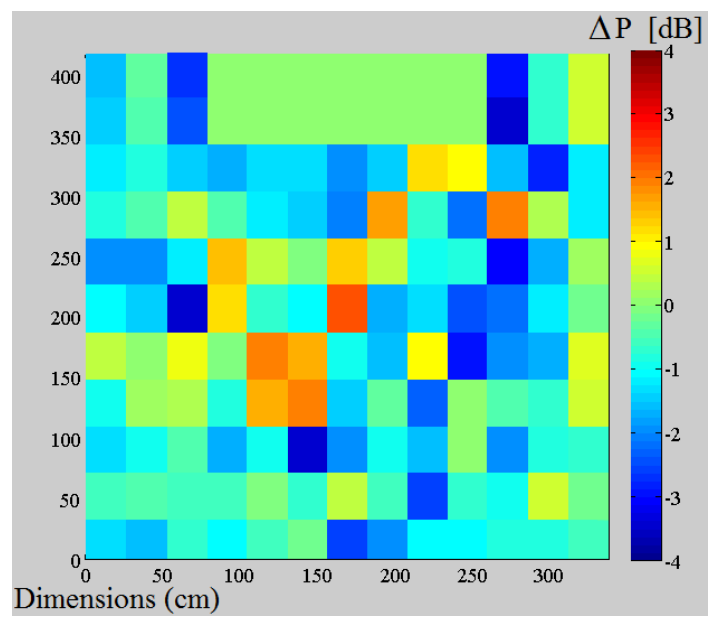

(b)

Figure 9: Difference of path loss $(\mathrm{dB})$ between measurements results and:

(a) Ray Tracing results (b) FDTD results.

The difference between Ray Tracing results and measurements results can be seen from -23 to $15 \mathrm{~dB}$, and the difference between FDTD results and measurements results can be seen from -3 to $2.5 \mathrm{~dB}$.

\section{PERFORMANCES OF METHODS}

In our study, we used for modeling with FDTD code one computer which has 8 processors and $32 \mathrm{~GB}$ of memory (RAM); the computation time was about 37 minutes. This simulation need $2.25 \mathrm{~GB}$ of RAM and the storage space on the hard disk was about $20 \mathrm{MB}$.
For the simulation with Ray Tracing, we used a powerful machine (16 processors, 96 GB RAM); the computational time was about 1 hour and 38 minutes. The results of this study show a big difference of capability to modeling electromagnetic propagation in complex environment between the FDTD code and Ray Tracing.

\section{CONCLUSION}

A rigorous electromagnetic indoor propagation modeling based on the FDTD (3D) method is presented in this paper taking into account the presence of various obstacles. The Ray Tracing method is used in order to compare, with FDTD code, the capability of modeling electromagnetic propagation in complex environments.

Numerical results are compared with measurements; a great capability of FDTD code to modeling with accuracy this kind of environment is noticed. The computational performance efficiency of these methods is also discussed.

The actual version of the FDTD code take into account the presence of human body, the characteristics of antenna should be integrated in the future to get more accuracy.

\section{References}

[1] Z. Sayegh, M. Latrach, F. Costen, W. Abdouni, G. El Zein, G. Zaharia, "Antenna Radiation in Typical Office Environment: Theoretical Modeling and Measurements", Computer Modeling and Simulation (EMS), 2012 Sixth UKSim/AMSS European Symposium on Mathematical Modelling and Computer Simulation, 14-16 Nov. 2012, pp. 433-438.

[2] Z. Sayegh, M. Latrach, F. Costen, W. Abdouni, G. El Zein, G. Zaharia, "Modélisation de la Propagation Electromagnétique dans un Environnement de Type Bureau à 2.4 GHz",Journées Nationales Microondes JNM,Paris, France, 15-17 May 2013.

[3] Allen Taflove, Susan C. Hagness, Computational Electrodynamics: The finite-difference timedomain method, Artech House, Norwood, 2005.

[4] Taflove A and Brodwin M, "Numerical solution of steady state electromagnetic scattering problems using the time dependent Maxwell's equations", IEEE MTT, Aug. 1975, pp. 623-630.

[5] Perambur S. Neeakanta, Theresa Kishkan, Rajeswari Chaterjee, "Antennas for Information Super Skyways: An Exposition on Outdoor and Indoor Wireless Antennas", Reaserch Studies Press Ltd, pp. 223, 1st edition (December 1, 2002). 